\title{
High Level Reduction Technique for Multiway Decision Graphs Based Model Checking
}

\author{
Ghiath Al Sammane, Sa'ed Abed and Otmane Ait Mohamed \\ Department of Electrical and Computer Engineering \\ Concordia University \\ 1455 de Maisonneuve \\ Montreal, Quebec, H3G 1M8, Canada \\ \{sammane, s_abed, ait\}@ece.concordia.ca
}

\begin{abstract}
Multiway Decision Graphs (MDGs) represent and manipulate a subset of first-order logic formulae suitable for model checking of large data path circuits. Due to the presence of abstract variables, existing reduction algorithms that is defined on symbolic model checking with BDD cannot be used with MDG. In this paper we propose a technique to construct a reduced MDG model for circuits described at algorithmic level in VHDL. The simplified model can be obtained using a high level symbolic simulator called TheoSim, and by running an appropriate symbolic simulation patterns. Then, the actual proof of a temporal MDG formula will be generated. We support our reduction technique by experimental results executed on benchmark properties.
\end{abstract}

Keywords: Model-checking, Symbolic Simulation, Behavioral Models

\section{INTRODUCTION}

The current context of systems-on-a-chip design involves the use of large architectural blocks, such as CPU cores, complex operators, and parameterized memory modules. These components are typically specified with algorithms written in high level languages like $\mathrm{C}_{++}$and Matlab, and their behavioral description extensively validated, mainly by the simulation of test cases.

At this stage, theorem-proving techniques are gaining attention, but their use is still considered difficult and time consuming. Model checking stills the automatic formal verification technique that is preferred for industrial flows. It aims by exploring the reachable state space of a model to verify that an implementation satisfies a specification [1]. Binary Decision Diagram (BDD) [2] is the canonical representation for Boolean functions that is used initially by model checker as an efficient encoding for the state space at the Boolean level.

In fact, efficient equivalence checking tools are available at the the Register Transfer Level (RTL) and below. New, property checking of logic-level control parts is gaining steam, with the combination of BDD's, Satisfiability (SAT) solvers, and simulation. In order to use these existing tools and methods directly on system level models a synthesis step is needed. Then, the space state becomes, in most cases, extremely large and that leads to the problem of state-explosion, which limits the applications of to small-to-medium systems.

Multiway Decision Graphs (MDGs) [3] is an alternative that extended BDD and SAT based model checking. MDG represents and manipulate a subset of first-order logic formulae suitable for large system level circuits. With MDGs, a data value is represented by a single variable of an abstract type and operations on data are represented in terms of an uninterpreted functions. The MDG operations and verification procedures are packaged as MDG tools and implemented 
in Prolog [5] providing facilities for invariant checking, verification of combinational circuits, equivalence checking of two state machines and model checking.

Due to the presence of abstract variables, existing reduction algorithms which are defined on symbolic model checking with BDD cannot be used with MDG. In this paper we propose a simple but powerful technique to construct a reduced MDG model for circuits described at algorithmic level in VHDL. By using a high level symbolic simulator called TheoSim, and by running an appropriate symbolic simulation patterns before the actual proof of a temporal MDG formula, an important model simplification can be obtained. We support our reduction technique by experimental results executed on benchmark properties.

The organization of this paper is as follows: Section 2 gives some preliminaries on MDG system and TheoSim, respectively. The main contribution of the paper describing the reduction technique is presented in Section 3. Section 4 discusses experimental results of applying our reduction methodology. Section 5 and 6 , review the related work in the area and discuss the possibilities for future research directions, respectively.

\section{PRELIMINARIES}

\subsection{The Multiway Decision Graph}

Multiway Decision Graph (MDG) is a graph representation of a class of quantifier-free and negation-free first-order many sorted formulas, called Directed Formulae, (DFs) [3]. DFs can represent the transition and output relations of a state of machine, as well as the set of states. As in ordinary many-sorted First Order Logic (FOL), terms are made out of sorts, constants, variables, and function symbols. Two kinds of sorts are distinguished: concrete and abstract:

- Concrete sort is equipped with finite enumerations, lists of individual constants. Concrete sorts is used to represent control signals.

- Abstract sort has no enumeration available. It uses first order terms to represent data signals.

Figure 1 shows two examples $\mathrm{G} 0$ and G1. In G0, $x$ is a variable of the concrete sort $[0,1,2,3]$. By contrast, in $\mathrm{G} 1 x$ is a variable of abstract sort where $\alpha, \beta$ and $f(\theta)$ are abstract terms.

G0

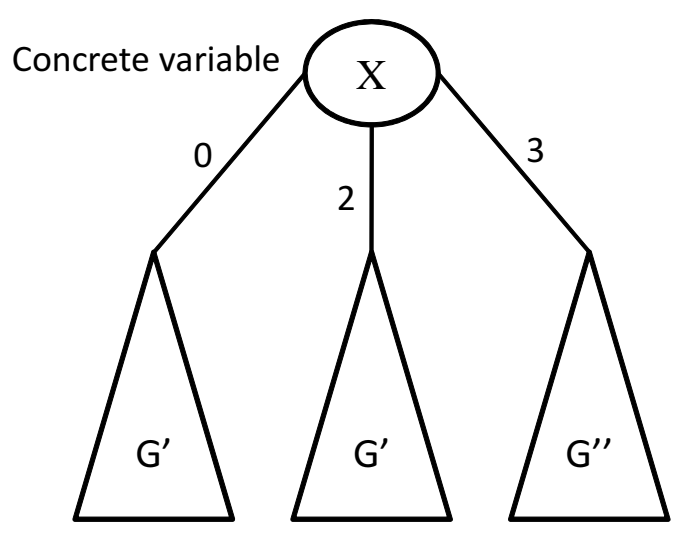

G1

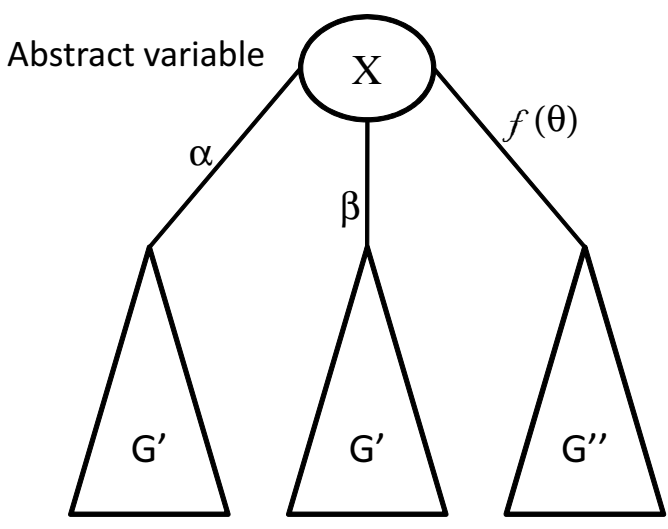

FIGURE 1: Example of Multiway Decision Graphs Structure

MDG terms are well formed first-order term. Let $\mathcal{F}$ be a set of function symbol and $\mathcal{V}$ a set of variables. We denote the set of terms freely generated from $\mathcal{F}$ and $\mathcal{V}$ by $\mathcal{T}(\mathcal{F}, \mathcal{V})$. The syntax of a Directed formulae DF is then given by the grammar below [4]. The underline is used to 
differentiate between the concrete and abstract.

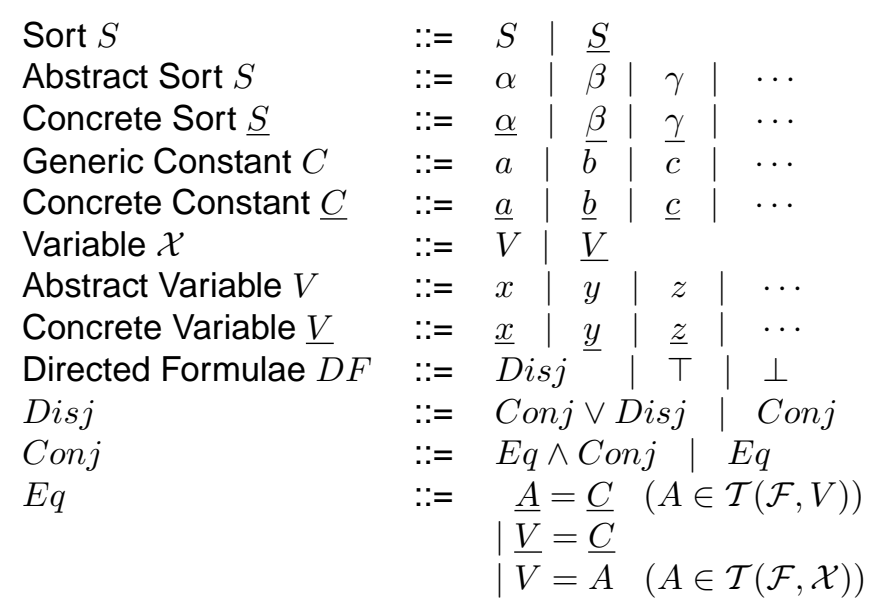

Directed Formulae are always disjunction of conjunctions of equations or True or False (terminal case). The Disj can be disjunction of conjuncts or a conjunct only. Also, the conjunction Conj is defined to be an equation only Eq or a conjunction of at least two equations. Atomic formulae are the equations, generated by the clause Eq. The equation can be the equality of concrete term and an individual constant, the equality of a concrete variable and an individual constant, or the equality of an abstract variable and an abstract term.

Directed Formulae are used for two purposes: to represent sets (viz. sets of states as well as sets of input vectors and output vectors) and to represent relations (viz. the transition and output relations).

\subsection{The MDG-Tool}

The MDG operations and verification procedures are packaged as MDG tools and implemented in Prolog [5]. The MDG tools provide facilities for invariant checking, verification of combinational circuits, sequential verification, equivalence checking of two state machines and model checking. It is based on a limited nesting of temporal operators (other than $\mathbf{X}$ ) where existential abstraction and a Prune-by-Subsumption are applied to compute a merged set of reachable states.

The input language of the MDG tool is a Prolog-style hardware description language (MDG$H D L$ ) [6], which supports structural specification, behavioral specification or a mixture of both. A structural specification is usually a netlist of components connected by signals, and a behavioral specification is given by a tabular representation of transition/output relations or a truth table.

In MDG model checking, the system is expressed as an Abstract State Machines (ASM) and the properties to be verified are expressed by formulas in $\mathcal{L}_{M D G} . \mathcal{L}_{M D G}$ atomic formulas are Boolean constants (True and False), or equations of the form $t_{1}=t_{2}$, where $t_{1}$ is an ASM variable (input, output or state variable) and $t_{2}$ is either an ASM system variable, an individual constant, an ordinary variable or a function of ordinary variables. Ordinary variables are defined to memorize the values of the system variables in the current state.

Any $\mathcal{L}_{M D G}$ formula is built using the basic operator Next_let_formulae, in which only the temporal operator $\mathbf{X}$ (next time) is allowed. The structure of Next_let_formulae is defined as follows (see [4] for more details):

- Each atomic formula is a Next_let_formulae;

- If $p, q$ are atomic formula then the following are Next_let_formulas:

- ! $p($ not $p), p \& q(p$ and $q) p \mid q(p$ or $q), p \rightarrow q$ ( $p$ implies $q)$

- Xp (next-time $p)$

- LET $(v=t)$ IN $p$, where $t$ is a system variable and $v$ an ordinary variable. 
Model checking of a property $\mathrm{p}$ in $\mathcal{L}_{M D G}$ on an $A S M M$ is carried out by building ASMs for sub-formulae containing only the temporal operator $\mathbf{X}$. Then, these additional ASMs are composed with $M$. Thus, a simpler property is checked on the composite machine [7].

Figure 2 shows the structure of the MDG-tool. In order to verify designs with the tool, we first need to specify the design in MDG-HDL (design specification and design implementation). Moreover, an algebraic specification is to be given to declare sorts, function types, and generic constants that are used in the MDG-HDL description. Rewrite rules that are needed to interpret function symbols should be provided here as well. Like for ROBDDs, a symbol order according to which the MDG is built should be provided by the user. However, there are some requirements on the node ordering of abstract variables and cross-operators (but not for concrete variables). This symbol order can affect critically the size of the generated MDG. Otherwise, MDG uses automatic dynamic ordering.

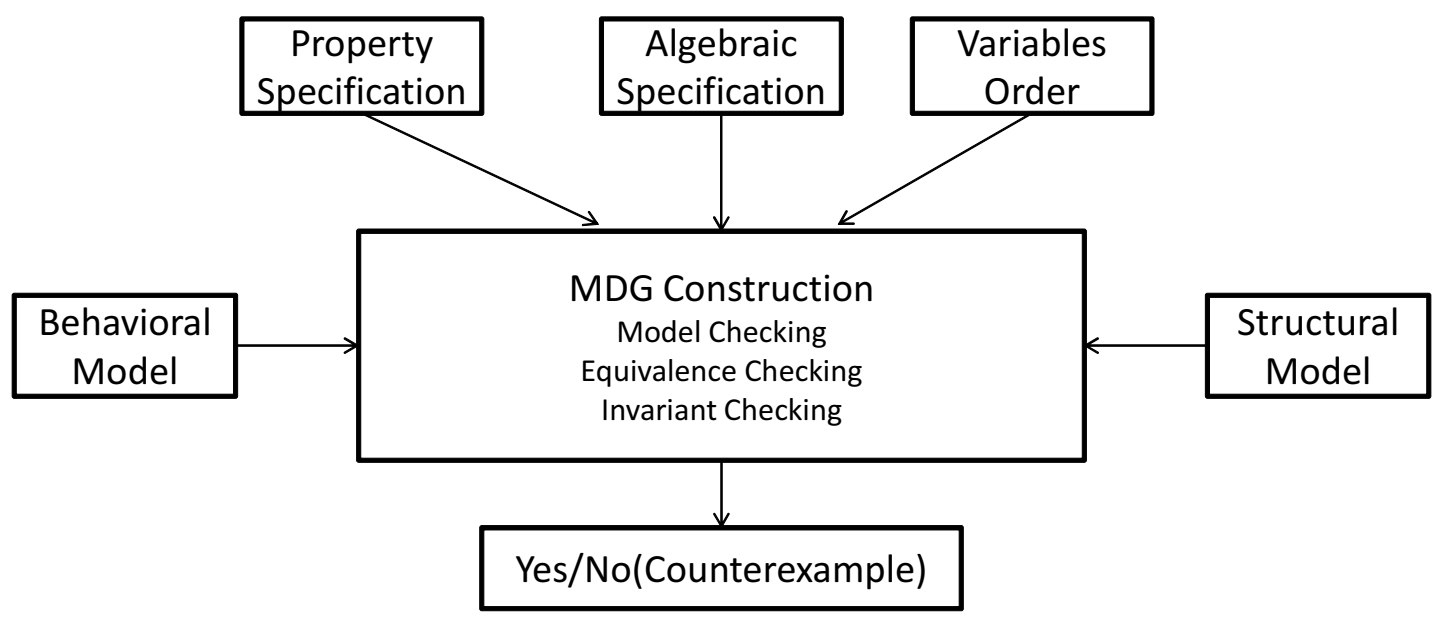

FIGURE 2: The Structure of the MDG-tool

\subsection{TheoSim}

TheoSim is a high level symbolic simulator based on the System of Recurrence Equations (SRE) model and implemented in the Computer Algebra System Mathematica [10]. It makes use of Mathematica symbolic engine and its built-in algebraic simplification rules.

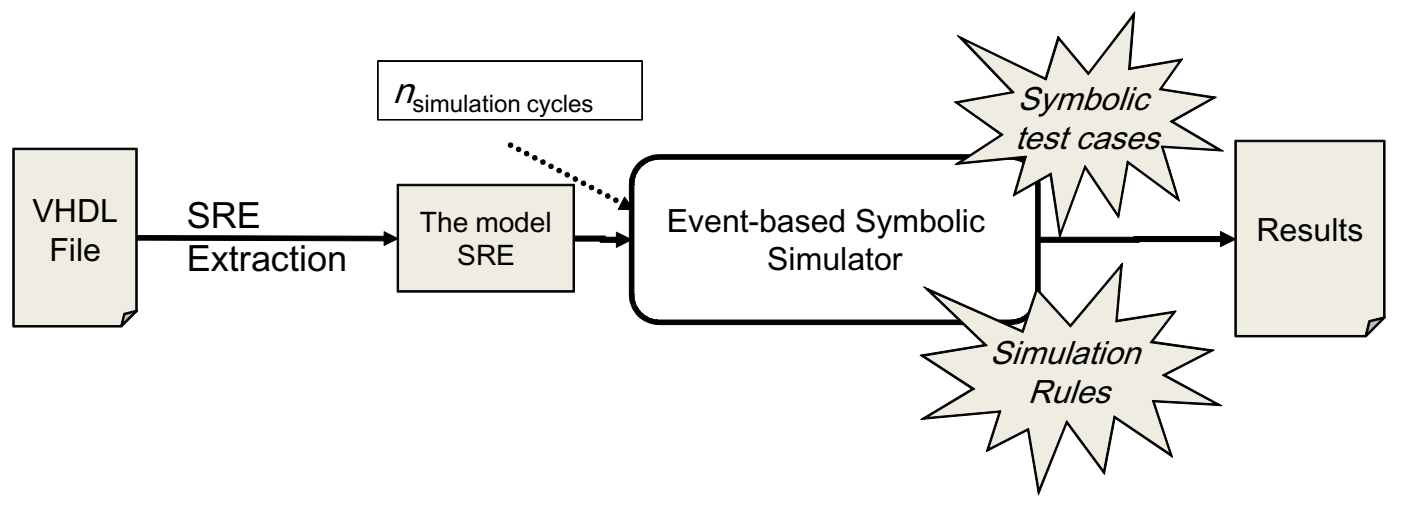

FIGURE 3: Overview of TheoSim

As shown in Figure 3, TheoSim provide a compiler that extracts automatically the SRE model from a VHDL behavioral design, an automatic generator of simulation patterns, and an event driven symbolic simulator written in Mathematica 


\subsubsection{The System of Recurrence Equations (SRE)}

A System of Recurrence Equations (SRE) is a 5-tuple :

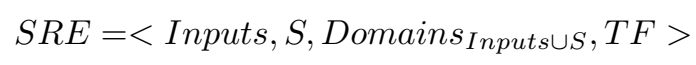

- All the elements in Inputs and in $S$ are represented as time variables of the form $X_{i}(n)$.

- Inputs $=\left\{I_{1}(t), I_{2}(t), \ldots, I_{n}(t)\right\}$ is the set of input signals of the system

- $S=$ Locals $\cup$ Outputs $=\left\{S_{1}(t), S_{2}(t), \ldots, S_{m}(t)\right\}$, is the set of simulated objects. With:

- Locals is the set of internal signal and variables

- Outputs is the set of output signals of the system

- Domain Inputs $\cup S_{S}$ is the set that contains the mathematical domain of each element in Inputs and $S$.

- $T F$ is the transition function that describes the behavior of the system at the end of simulation cycle $t$; written as set of recurrence equations, one equation for each elements in $S$ : For a circuit that contains $m$ objects (signals + variables), we extract automatically a set of $m$ Equations: $\left\{X_{i}(t)\right\}_{0<i \leq m}$ where, $X_{i}(t)$ is the value of the object $i$ at time $t$ given as a recurrence equation:

$$
X_{i}(t)=f_{i}\left(X_{j}(t-\gamma)\right) \quad \text { with } m, i, j, \gamma \in \mathbb{N} \text {, and }(0<i \leq m, 0<j \leq m, 0<\gamma), \forall t \in \mathbb{N}
$$

These equations form an algebraic partitioned version for the transition relation of the circuit. It is analyzed using a cone of influence based algorithm [9], which means that all variables that do not influence the value of the object $X_{i}(t)$ are eliminated from the expression of the function $f$. The function $f$ is normalized using a generalized if-then-else expressions.

For example, considering the behavioral counter described in VHDL:

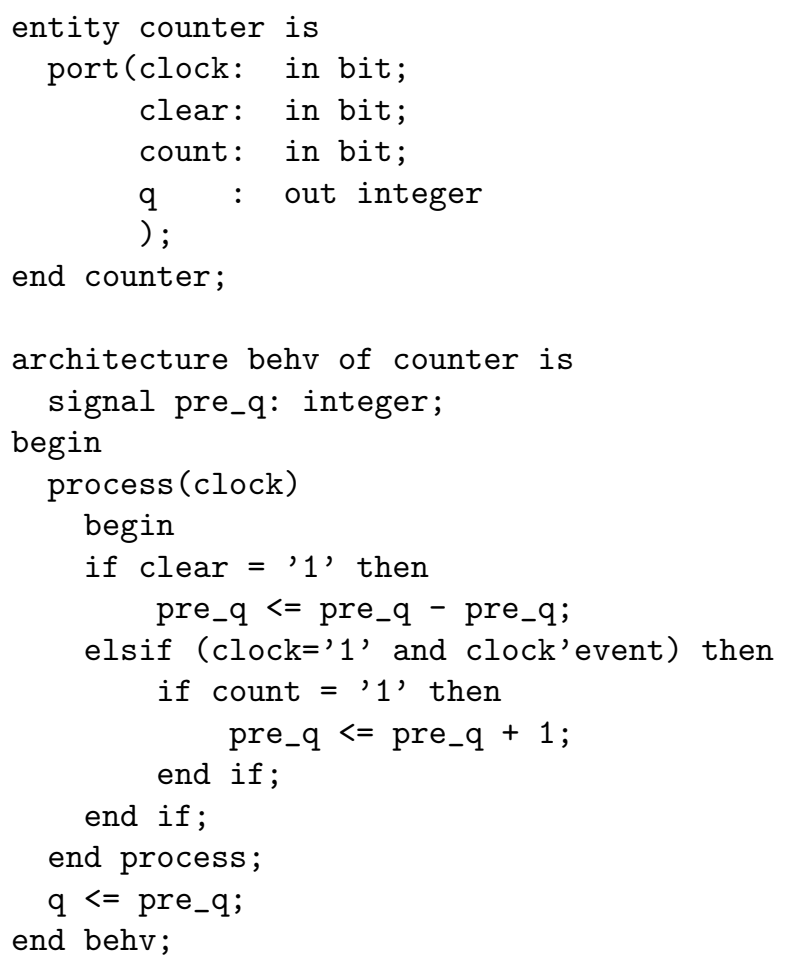

The SRE of this counter is :

Inputs $=\{\operatorname{clock}(t), \operatorname{clear}(t), \operatorname{count}(t)\}$,

Locals $=\{$ pre_ $q(t)\}$,

Outputs $=\{q(t)\}$,

Domains $_{\text {Inputs } \cup S}=\left\{\operatorname{clock}(t) \in \mathbb{B}, \operatorname{clear}(t) \in \mathbb{B}, \operatorname{count}(t) \in \mathbb{B}\right.$, pre $\left.\_(t) \in \mathbb{Z}, q(t) \in \mathbb{Z}\right\}$ 
The set of recurrence equations of the design contains the equation of $\left(q(t+1)\right.$ and pre $\left._{-} q(t+1)\right)$ : pre_q $(t+1)=$

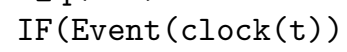

\subsubsection{The Event Based Symbolic Simulator}

Within TheoSim, the VHDL simulation algorithm is applied on the SRE of the circuit for a fixed number of simulation cycles $n$ given by the designer. During the simulation, a set of symbolic simulation patterns $\Delta$ is applied along with a set of simplification rules $\mathcal{R}$ that contains four kinds of rewriting rules:

$$
\mathcal{R}=R_{\text {Math }} \cup R_{\text {Logic }} \cup R_{I F} \cup R_{E q}
$$

Polynomial symbolic expressions $R_{M a t h}$ : are built-in rules intended for the simplification of polynomial expressions $\left(\mathbb{R}^{n}[x]\right)$.

Logical symbolic expressions $R_{\text {Logic }}$ : are rules intended for the simplification of Boolean expressions and to eliminate obvious ones like $(\operatorname{and}(a, a) \rightarrow a)$ and $(\operatorname{not}(\operatorname{not}(a)) \rightarrow a)$.

If-formula expressions $R_{I F}$ : are rules intended for the simplification of computations over if-then-else expression. The definition and properties of the $I F$ function, like reduction and distribution, are used (see [11] for more details):

- IF Reduction: $\operatorname{IF}(x, y, y) \rightarrow y$

- IF Distribution: $f\left(A_{1}, \ldots, I F(x, y, z), \ldots, A_{n}\right) \rightarrow \operatorname{IF}\left(x, f\left(A_{1}, \ldots, y, \ldots, A_{n}\right), f\left(A_{1}, \ldots, z, \ldots, A_{n}\right)\right)$

Equation rules: $R_{E q}$ are resulted from converting an SRE into a set of substitution rules.

The algorithmic details and the convergence of the rewriting algorithm is discussed in [8]. The symbolic simulation patterns $\Delta$ can be considered as a set of special assignment for the Inputs of the circuit at each $t$ and a special initialization sequence for the internal registers.

A symbolic simulation step takes values of the simulation patterns $\Delta$ at time $t$ and the set of simplification rules $\mathcal{R}$ and then applies them on the SRE until the achievement of a fixed point (FP).

$$
\text { SymSim_Step }\left(\left\{X_{i}(t)\right\}_{0<i \leq m}, \Delta, t\right)=\text { Replace_Until_FP }\left(\left\{X_{i}(t)\right\}_{0<i \leq m}, \mathcal{R}, \Delta, t\right)
$$


The result of the symbolic simulation depends on the nature of patterns $\Delta$ that can vary from a sequence of numerical values to a sequence of uninterpreted functions. In fact, the efficiency of our reduction technique relies on the good choice of $\Delta$ which can reduces the recurrence equations of the system.

\section{THE REDUCTION TECHNIQUE}

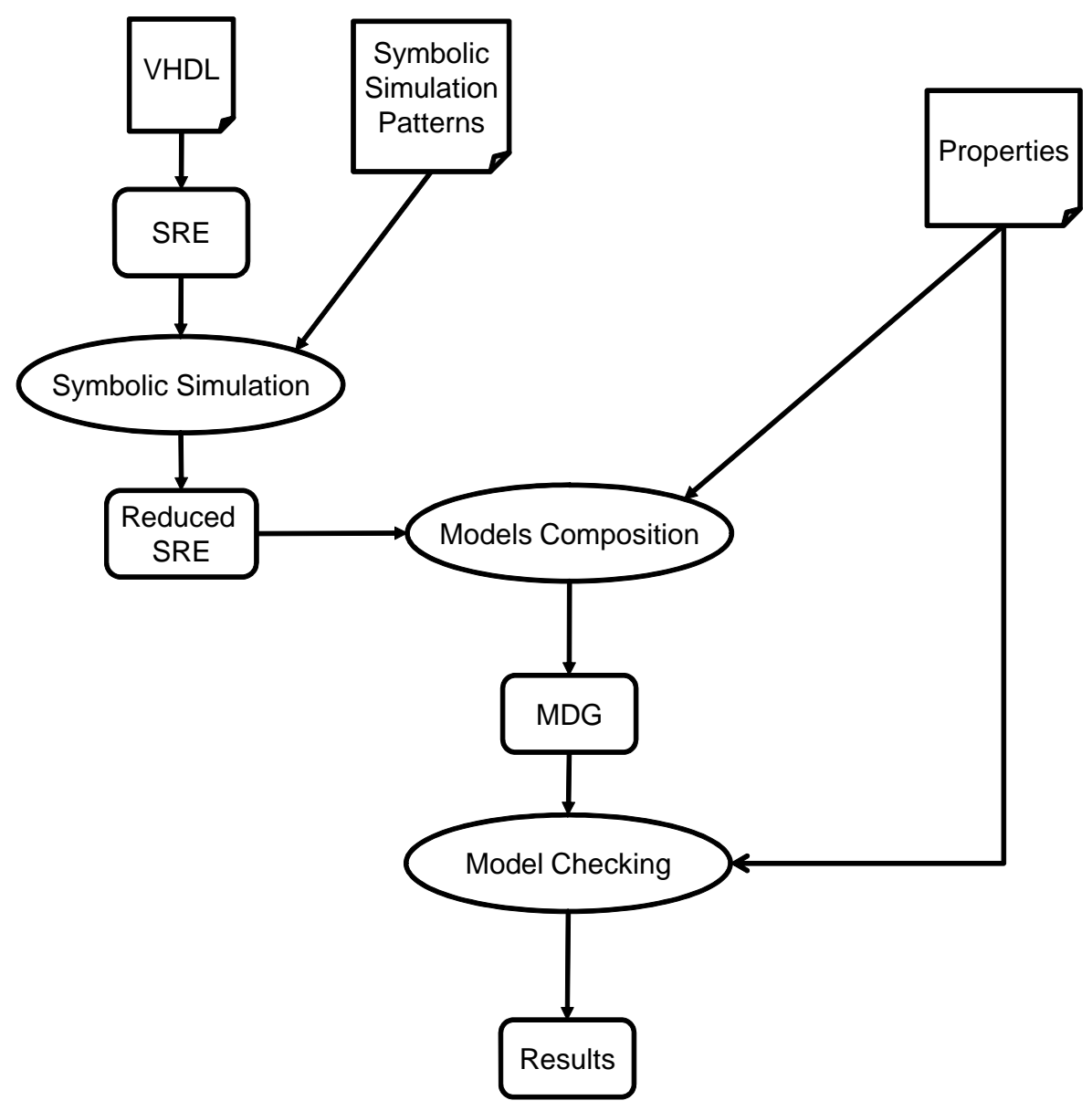

FIGURE 4: Overview of the Reduction Methodology

We start with a circuit design written in VHDL and a set of properties written in $\mathcal{L}_{M D G}$. As shown in Figure 4, we extract form the VHDL design a mathematical model in terms of a System of Recurrence Equations (SRE). From the properties, we write symbolic simulation patterns that are input along with the SRE model to a high level symbolic simulator. The reduction is done by applying the simulation patterns on the SRE model in order to obtain a reduced one. The next step, we compose the reduced SRE model with the $\mathcal{L}_{M D G}$ properties and we extract the reduced MDG. The formal verification is performed then on this resulted reduced MDG using the existing MDG package.

\subsection{Defining the Symbolic Simulation Patterns}

We provide a systematic strategy that does not need any expertise in the symbolic simulator or any knowledge of the implementation under verification. Only the specification of the circuit (expresses as a set of properties) is needed. Our reduction is inspired from [12] but we generalize it using SRE and MDG. 


\subsubsection{Synchronization detection}

Some complex System-on-Chip contain multiple synchronization clocks Clock $_{1}$, Clock $_{2}, \ldots$, Clock $_{m}$. Usually, the relation between these clocks has a periodical behavior given by the specifications. One reduction scenario is to assign numerical values for these internal clocks and to compute a reduced model where the symbolic expressions of the internal clocks are eliminated.

\subsubsection{Functional partitioning}

Even if the design of digital circuit is modular, it is rare that the structural design corresponds to a particular property. Thus, we cannot apply a modular verification approach. Using symbolic simulation, we can prune the transition relation using functional specifications depending on the property that we want to verify. The idea is to assign values to the control signals, one by one, depending on the tested operating mode. The result will be the elimination of symbolic expressions of non activated functional blocks and reducing the model to the activated one.

However, this assignment freeze the circuit in the selected operating mode. In order to prove the correctness of the circuit under all operation modes we need to split cases and to compute several reduced models. The model checking of the system, should be done on all the reduced models. The efficiency of this case splitting relies on the fact that the model checking time grows exponentially with the complexity of the circuit and the sum of exponentials is much less than the exponential of the sum.

\subsubsection{RESET elimination}

All digital circuits contain an initialization inputs noted as RESET. Practically, the interesting properties are not in the initialization phase of the circuit. We eliminate the RESET by activating it for a finite amount of time and then it should be deactivated.

\subsection{Computing the Reduced SRE}

We simulate the system for several simulation steps using the function SymSim_Step defined above. The simulation algorithm aims to reduce the SRE model by applying $\Delta$ as shown in Algorithm 1.

Line 1 first initialize the simulation time $t$ to $t_{0}$ (equal to zero in most cases). The purpose of line 2 and 3 is to store the initial SRE before applying the algorithm. The variables $\phi$ and $\varphi$ denote the reduced SRE and the initial SRE, respectively. Lines 4-7 repeatedly execute a symbolic simulation for $\mathrm{k}$ steps by applying $\Delta$ at the SRE. The algorithm returns the reduced SRE and the initial SRE (line 8-9). This equivalent to a new SRE where the time variable is changed to $T=t_{0}+k$. This reduced SRE will be used for MDG model-checking.

\subsection{Translating the Reduced SRE to MDG}

The reduced SRE is translated to MDG by three steps:

1. First, we introduce extra variables that will encode the time instance of a variables $X_{i}$ and $X_{j}$ in the equation $X_{i}(t)=f_{i}\left(X_{j}(t-\gamma)\right)$.

For example, the equation $X(t)=X(t-1)+1$ is encoded as $X^{\prime}=X+1$.

2. In the second step, we translate any function between the operators in the right hand side of the equation to uninterpreted functions representation. For example, $X^{\prime}=X+1$ become $X^{\prime}=\operatorname{Plus}(X, 1)$.

3. Finally, an if-then-else expression of the form $X=$ $I F$ (condition, then_branch,else_branch) is translated into a set of equalities of form: 


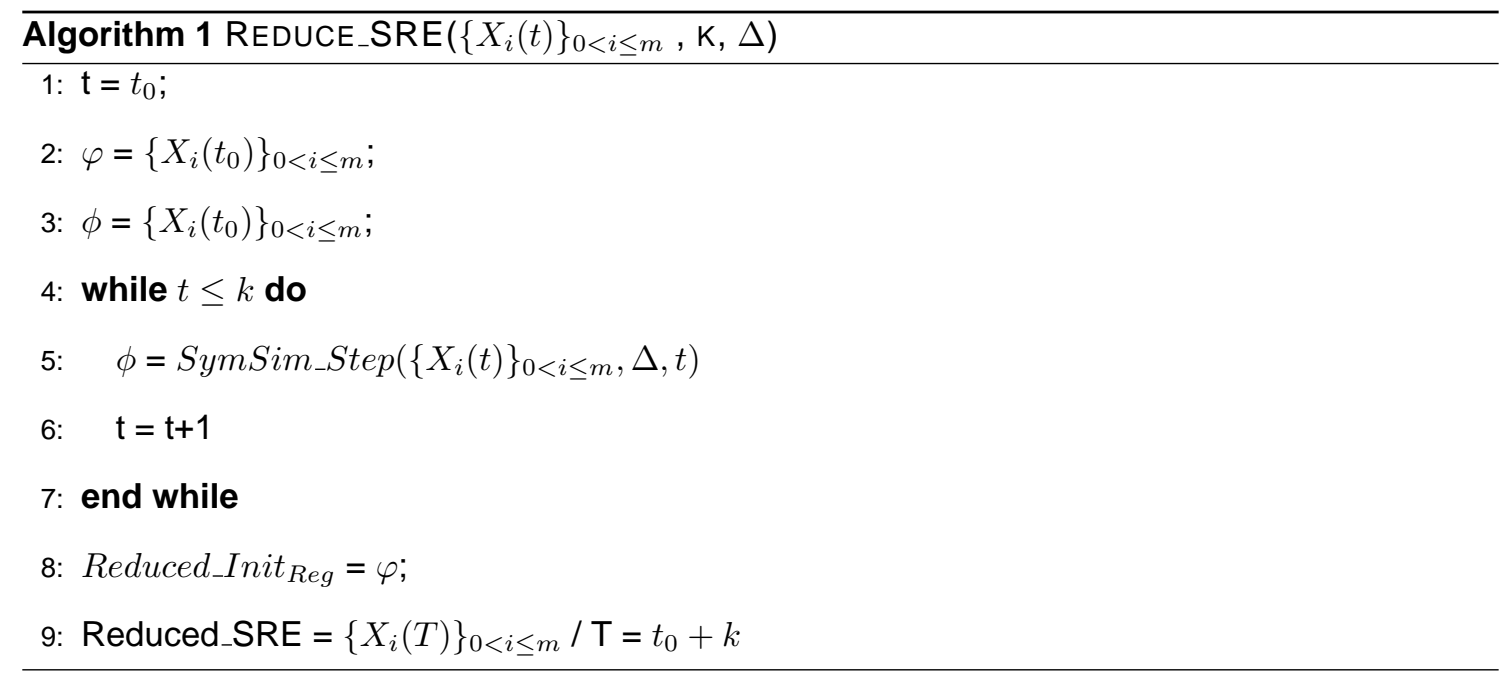

$($ condition $\wedge(X=$ then_branch $)) \vee(\neg$ condition $\wedge(X=$ else_branch $))$.

\subsection{Comparing with Cone of Influence}

The reduction obtained is different from reduction obtained with the cone of influence algorithm [9]. To illustrate this difference, we consider a basic RAM element defined at behavior level:

Inputs:\{reset, chip_select, add, data, output_enable \}

Outputs: $\{$ output $\}$

Registers: $\{r e g[0] \ldots r e g[n]\}$

if chip_select= 1 then

Writing Process

if reset $=1$ then

reg[add $]=0$

else if (read_write $=1$ ) and (output_enable $=0$ ) then reg[add] $=$ data

end if

Reading Process

if (read_write $=0$ ) and (output_enable $=1$ ) then (output=reg[add])

end if

end if

The transition relation for that circuit is the conjunction of both transition relations of the read and the write operations, in DF that will be written as follows: 
Read_Tr $r_{D F}=($ chip_select $=1) \wedge($ read_write $=0) \wedge($ output_enable $=1) \wedge($ output $=$ reg $[$ add $])$

Write_Tr $r_{D F}=($ chip_select $=1) \wedge(($ reset $=1) \wedge($ reg $[$ add $]=0) \vee$

$($ reset $=0) \wedge($ read_write $=1) \wedge($ output_enable $=0) \wedge($ reg $[$ add $]=$ data $))$

$R A M_{-} T r_{D F}=R e a d \_T r_{D F} \wedge W r i t e_{-} T r_{D F}$

If we want to verify a property about reading on the memory, then it is useful to symbolically simulate the system with select function assigned to the read active, which eliminates the symbolic expression of the selection function from the symbolic expression of the property. Thus, we obtain:

$\operatorname{Reduced}\left(R A M \_T r_{D F}\right)=($ output $=\operatorname{reg}[$ add $])$

The transition relation consists of one clause compared to 4 clauses given by the cone of influence algorithm (as it will return Read_Tr).

\section{APPLICATION AND RESULTS}

\subsection{The Island Tunnel Controller}

The MDG tool has been demonstrated on the example of the Island Tunnel Controller in [13], which was originally introduced by Fisler and Johnson [14]. The ITC controls the traffic lights at both ends of a tunnel based on the information collected by sensors installed at both ends of the tunnel: there is one lane tunnel connecting the mainland to an island. At each end of the tunnel, there is a traffic light. There are four sensors for detecting the presence of cars. It is assumed that all cars are finite in length, that no car gets stuck in the tunnel, that cars do not exit the tunnel before entering the tunnel, that cars do not leave the tunnel entrance without traveling through the tunnel, and that there is sufficient distance between two cars such that the sensors can distinguish the cars.

The ITC controller for the traffic lights at a one lane tunnel connecting the mainland to a small island as depicted in Figure 5. There is a traffic light at each end of the tunnel; there are also four sensors for detecting the presence of vehicles: one at tunnel entrance on the island side (ie), one at tunnel exit on the island side (ix), one at tunnel entrance on the mainland side (me), and one at tunnel exit on the mainland side $(m x)$.

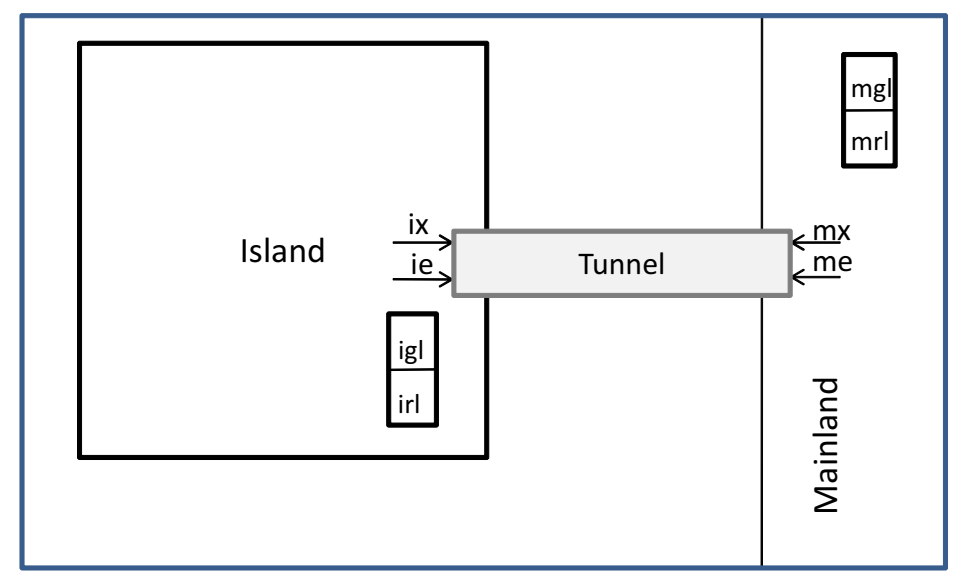

FIGURE 5: Island Tunnel Controller Structure

The ITC is composed of five modules: The Island Light Controller, the Tunnel Controller, the Mainland Light Controller, the Island Counter and the Tunnel Counter (refer to [14] for the state 
transition diagrams of each component). The Island Light Controller (ILC) has four states: green, entering, red and exiting. The outputs igl and irl control the green and red lights on the island side respectively; iu indicates that the cars from the island side are currently occupying the tunnel, and ir indicates that ILC is requesting the tunnel. The input iy requests the ILC to release control of the tunnel, and ig grants control of the tunnel from the island side as shown in Figure 6. A similar set of signals is defined for the Mainland Light Controller (MLC).

The Tunnel Counter (TC) processes the requests for access issued by ILC and MLC. The Island Counter and the Tunnel Counter keep track of the car's number currently on the island and in the tunnel, respectively. For the tunnel controller, the counter tc is increased by 1 depending on $t c+$ or decremented by 1 depending on tc- unless it is already 0 . The Island Counter operates in a similar way, except that increment and decrement depend on ic+ and ic-, respectively: one for the island lights, one for the mainland lights, and one tunnel controller that processes the requests for access issued by the other two controllers.

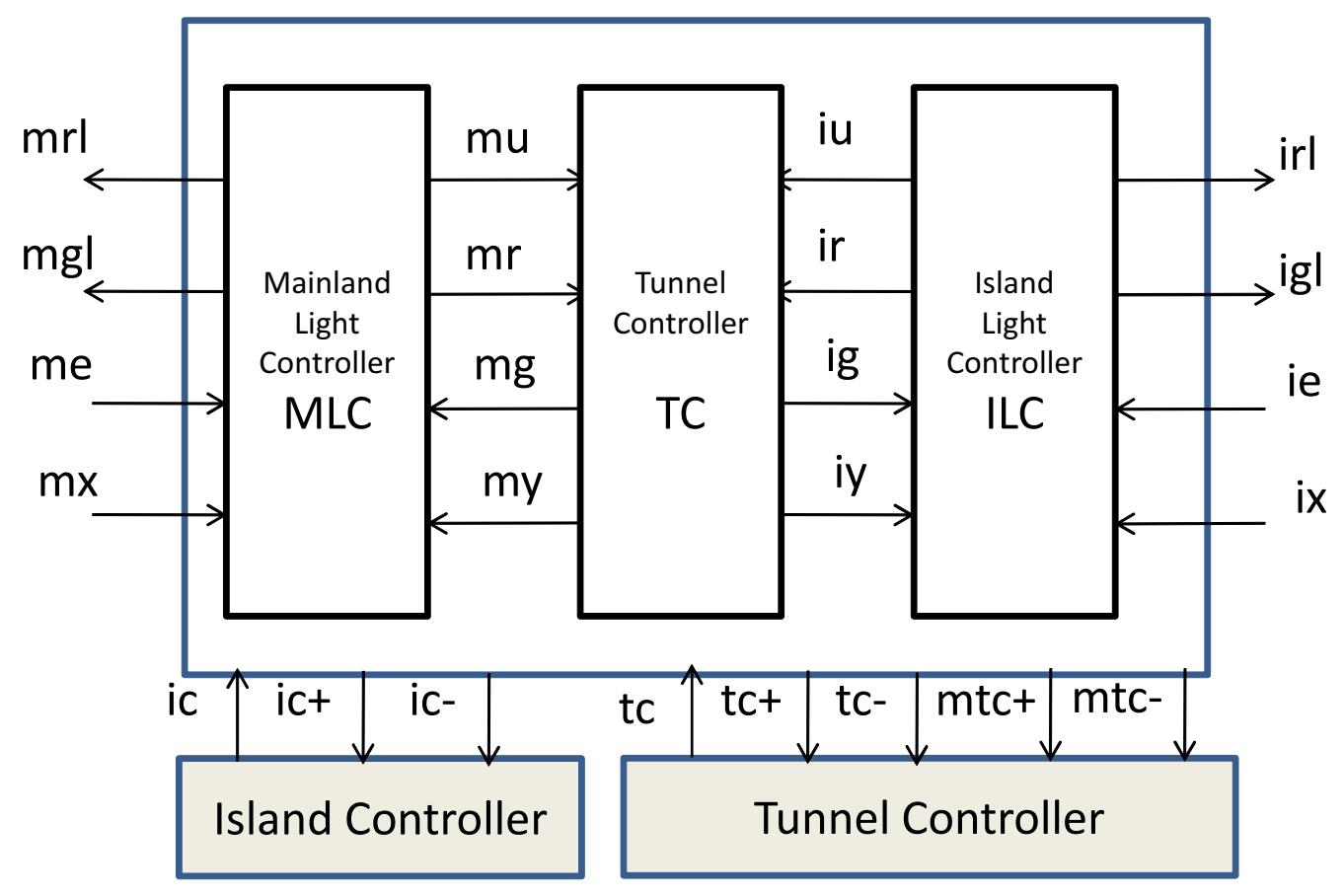

FIGURE 6: Island Tunnel Controller Structure

We would like to establish that the ITC has at least the following properties:

- Property 1: Cars never travel both directions in the tunnel at the same time.

- Property 2: Access to the tunnel is not granted until the tunnel is empty.

- Property 3: Lights don't turn green until access is granted.

- Property 4: Requests for the tunnel are eventually granted.

- Property 5: There are never more than $m$ cars on the island.

\subsection{Experimental Results}

We take the same case study and we consider the ITC with its properties as a benchmark in order to measure the performance of our approach. Table 1 compares the verification results with and without reduction for five properties, run on an Ultra2 Sun workstation with $296 \mathrm{MHz}$ CPU and 
TABLE 1: Comparing Model Checking Results with \& without Reduction

\begin{tabular}{c||c|c|c||c|c|c}
\hline \hline \multirow{2}{*}{$\begin{array}{c}\text { Benchmark } \\
\text { Properties }\end{array}$} & \multicolumn{3}{c||}{ Without Reduction } & \multicolumn{3}{c}{ With Reduction } \\
\cline { 2 - 7 } & Time & Memory & Nodes & Time & Memory & Nodes \\
\hline \hline P1 & 87.41 & 45.5 & 123080 & 86.99 & 43.4 & 121060 \\
\hline P2 & 87.41 & 0.56 & 263 & 1.12 & 0.4 & 211 \\
\hline P3 & 87.56 & 48.7 & 123085 & 14.46 & 6.82 & 12292 \\
\hline P4 & 88.77 & 48.8 & 123082 & 1.02 & 0.06 & 16 \\
\hline P5 & 89.87 & 47.5 & 123080 & 1.15 & 0.58 & 241 \\
\hline \hline Average & 88.2 & 38.22 & 98518 & 20.95 & 10,25 & 26764 \\
\hline \hline
\end{tabular}

$768 \mathrm{MB}$ memory. We give the CPU time measured in seconds and the memory measured in MB that are used in building the reduced machine and checking the property.

We remark that the reduction is influenced by the properties. The best gain in performance is obtained with property $\mathrm{P} 4$ where the time is reduced by 77 times the original one and the memory is reduced by a factor of 88 times. The worst case is the property $\mathrm{P} 1$ where the reduction algorithm is not profitable. In the case of property P1 the assumptions and the functionality tested needs several runs (when using case splitting). The sum of these runs is equal for this particular case to a single run without reduction. For $\mathrm{P} 4$, it was the other extremum where case splitting was really much more efficient.

These differences show the sensitivity of the reduction technique to the property verified. Despite these fluctuations, the average of the gain in performance is a factor of 4 which is considered as a good result in the case of model checking approaches.

\section{RELATED WORK AND DISCUSSION}

Compositional model checking [15] is a reduction strategy that aims at splitting a proof goal into several simple sub-goals, which are determined by choosing appropriate constrain functions for a subset of primary inputs of the design. From a behavioral point of view, this amounts to applying Algorithm 1 with parameter $\mathrm{k}$ set to 1 . The reduced model is constructed according to the user supplied constrained functions. The strategy we propose generalizes case splitting to a sequence of values of arbitrary length.

In the same direction, Hazelhurst et al. presented in [16] a hybrid approach relying on the use of two industrial tools, one for symbolic trajectory evaluation (STE) [17] and one for symbolic model-checking. STE performs user-supplied initialization sequences and produces a parametric representation of the reached states set. The result must be systematically converted into a characteristic function form, before it can be fed to the model-checker. The conversion process is expensive in the general case [16].

In [12], a model checking reduction by symbolic simulation is presented. This method has been implemented within the SMV model checker. By contrast to our technique these methods are applicable only at the Boolean level. However, our methodology is applicable at higher level of abstraction as it makes use of SRE based symbolic simulation.

In [18] an MDG reduction technique is proposed. A reduced abstract transition system is derived from the original ASM using only the transition relations of the so-called property dependent state variables VP of a property P; to be verified. This reduction technique is equivalent only to a cone of influence reduction. We propose further reductions that includes cone of influence as well. 


\section{CONCLUSION AND FUTURE WORK}

We have proposed a reduction technique for MDG model checking that uses a high level symbolic simulation. The symbolic simulation provides behavioral VHDL as an input language for MDG tools.

The symbolic simulator is based on an intermediate mathematica representation in terms of recurrence equations (SRE). A first reduction that based on cone of influence analysis is applied while extracting the SRE model from the VHDL file. Later, we have used the information in the properties to extract symbolic simulation patterns and applied on the model several strategies: functional partitioning, case splitting, and RESET elimination. The symbolic simulation for $k$ steps applies these strategies and provide a partial interpretation for the recurrence equations model which resulted in a reduced model. The originality of our reduction technique comes from applying rewriting based symbolic simulation. Thus, we obtain reduced models at high level of abstraction that involves integers and reals.

We have illustrated the gain in performance on benchmark properties recognized by the MDG community. However, the approach stills in its early stage. More experimentations are needed in order to identify the strength and the limits of the approach. Today, symbolic simulation patterns are written manually by the designer. Our future work concentrate on this issues in two directions:

1) consider properties written in the standard Property Specification Language.

2) automatically extracts the simulation patterns from the properties.

\section{REFERENCES}

[1] E. M. Clarke, O. Grumberg, and D. E. Long. Model Checking. In Nato ASI, vol. 152 of F, Springer-Verlag, 1996.

[2] R. Bryant. Graph-Based Algorithms for Boolean Function Manipulation. In IEEE Transactions in Computer Systems, 35(8): 677-691, August 1986.

[3] F. Corella, Z. Zhou, X. Song, M. Langevin, and E. Cerny. Multiway Decision Graphs for Automated Hardware Verification. In Formal Methods in System Design, 10(1): 7-46, 1997.

[4] O. Ait Mohamed, X. Song, and E. Cerny. On the non-termination of MDG-Based Abstract State Enumeration. In Theoretical Computer Science Journal, 1-3(300): 161-179,2003.

[5] W. Clocksin and C. Mellish. Programming in Prolog. Springer-Verlag, 3rd edition, 1987.

[6] Z. Zhou and N. Bouleric. MDG Tools (V1.0) User's Manual. D'IRO, University of Montreal, Canada, 1996.

[7] Ying Xu, Xiaoyu Song, Eduard Cerny, and Otmane Ait Mohamed. Model Checking for a FirstOrder Temporal Logic Using Multiway Decision Graphs (MDGs). In The Computer Journal, 47(1): 71-84, 2004.

[8] G. Al Sammane. Symbolic simulation of circuits described at algorithmic level, PhD thesis, Joseph Fourier University, Greoble, France, 2005, ISBN 2-84813-069-5.

[9] P. Cousot and R. Cousot. Abstract interpretation: a unified lattice model for static analysis of programs by construction or approximation of fixpoints. In Proc. of the 4th ACM SIGACTSIGPLAN Symposium on Principles of Programming Languages (Los Angeles, California, January, 1977).

[10] S. Wolfram, The Mathematica Book, Fifth Edition, Wolfram Media Inc., 2003.

[11] J. Strother Moore. Introduction to the OBDD Algorithm for the ATP Community, J. Autom. Reasoning, vol. 12, pp. 33-45, 1994.

[12] Dumitrescu, E.; Borrione, D., Symbolic simulation as a simplifying strategy for SoC verification, The 3rd IEEE International Workshop on System-on-Chip for Real-Time Applications, 2003.

[13] Z. Zhou, X. Song, S. Tahar, E. Cerny, F. Corella, and M. Langevin. Formal verification of the island tunnel controller using multiway decision graphs. In Formal Methods in Computer Aided Design (FMCAD), 1996.

[14] K. Fisler and S. Johnson. Integrating design and Verification Environments Through A Logic Supporting Hardware Diagrams. In Proc. of IFIP Conf. on Hardware Description Languages 
and their Applications (CHDL'95), Japan, August 1995.

[15] K. L. McMillan. Verification of infinite state systems by compositional model checking. Conf. on Correct Hardware Design and Verification Methods, 1999.

[16] S. Hazelhurst, O. Weissberg, G. Kamhi, and L. Fix, A Hybrid Verification Approach: Getting Deep into the Design, 39th Design Automation Conf., (DAC '02), June 2002, pp. 111-116.

[17] S Hazelhurst and C J Seger. Symbolic Trajectory Evaluation. In T Kropf, ed., Formal Hardware Verification: Methods and Systems in Comparison, LNCS 1287, pp. 3-79. SpringerVerlag, Berlin.

[18] Jin Hou; Cerny, E., Model reductions in MDG-based model checking, In the Proc. of 13th Annual IEEE International ASIC/SOC Conference, 2000. 